\title{
Intelligence profiles of Chinese school-aged boys with high-functioning ASD and ADHD
}

\author{
This article was published in the following Dove Press journal: \\ Neuropsychiatric Disease and Treatment \\ 15 June 2017 \\ Number of times this article has been viewed
}

Gaizhi Li, ${ }^{1, *}$ Wenqing Jiang, ${ }^{2, *}$

Yasong Du, ${ }^{2}$ Kathryn

Rossbach $^{3}$

'Shanghai Mental Health Center, Shanghai Jiao Tong University School of Medicine, Shanghai, People's Republic of China; ${ }^{2}$ Department of Child \& Adolescent Psychiatry, Shanghai Mental Health Center, Shanghai Jiao Tong University School of Medicine, Shanghai, People's Republic of China; ${ }^{3}$ Emory Autism Center, Emory University School of Medicine, Atlanta, GA, USA

*These authors contributed equally to this work

Video abstract

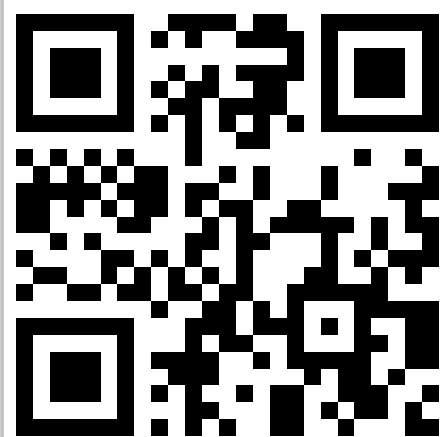

Point your SmartPhone at the code above. If you have a QR code reader the video abstract will appear. Or use:

http://youtu.be/x40ydnm_E3w

Correspondence: Yasong Du Department of Child \& Adolescent Psychiatry, Shanghai Mental Health Center, Shanghai Jiao Tong University School of Medicine, No 600 Wanping Nan Road, Xuhui, Shanghai 200030, People's Republic of China

Tel +862164387250 Ext 3420

Fax +862164387986

Email yasongdu@I63.com
Purpose: This study aimed to explore the intelligence profiles of Chinese school-aged boys with high-functioning autism spectrum disorder (HFASD) and attention-deficit/hyperactivity disorder (ADHD). Additionally, differences in intelligence quotient (IQ) between the HFASD group and the ADHD group were examined.

Patients and methods: Thirty-two boys with HFASD, 58 boys with ADHD, and 39 typically developing (TD) boys aged 6-16 years participated in this study. The ADHD group was divided into subgroups: ADHD-I (predominantly inattentive) and ADHD-C (combined type). (The ADHD-H [hyperactive] group was excluded because of small sample size). The Wechsler Intelligence Scale for Children-IV Chinese version was administered to every participant, and the FSIQ (Full-Scale IQ) score was used as the measure of IQ.

Results: Both boys with HFASD and ADHD (ADHD-I and ADHD-C) showed impairments in Processing Speed Index and FSIQ, as compared to the TD group. Lower Verbal Comprehension Index scores were found in the ASD and ADHD-I groups. Interestingly, Working Memory Index was only impaired in children with ADHD. Additionally, equivalent Perceptual Reasoning Index (PRI) scores were found among the HFASD, ADHD, and TD groups.

Conclusion: Results indicated that both children with ADHD and HFASD have difficulty in processing speed, which may be explained by these children having neurodevelopmental disorders. These results also indicated that working memory appears to only be impacted by having ADHD. Children with ASD are known to have language difficulties while children with ADHD typically display working memory deficits; thus, these findings were expected.

Keywords: autism spectrum disorder, attention-deficit/hyperactivity disorder, child, intelligence, IQ, WISC-IV Chinese version

\section{Plain language summary}

This study compared the intellectual differences of boys with high-functioning autism spectrum disorder (HFASD) and attention-deficit/hyperactivity disorder (ADHD) (subgroups included) with typically developing boys, using the Wechsler Intelligence Scale for Children-IV (WISC-IV) Chinese version. The WISC-IV Chinese version generates a Full-Scale Intelligence Quotient as well as four index scores, including the Verbal Comprehension Index, Perceptual Reasoning Index (PRI), Processing Speed Index, and Working Memory Index. Results indicated that both children with ADHD and HFASD had difficulty in processing speed, while working memory appears to only be impacted in boys with ADHD. PRI scores were equivalent among the HFASD, ADHD, and typically developing boys.

\section{Introduction}

According to the Diagnostic and Statistical Manual of Mental Disorders, Fifth Edition (DSM-5), autism spectrum disorder (ASD) is characterized by an impairment in social communication as well as displaying restrictive or repetitive patterns 
of thought or behavior. In the most recent statistics, ASD affects $\sim 1$ in 68 children. ${ }^{1}$ Further, about $1 / 3$ of children with ASD suffer from an intellectual disability. ${ }^{1}$ As mentioned by Lincoln, high-functioning ASD is defined by having an intelligence quotient (IQ) above $70 .^{2}$ These individuals would not have any sort of intellectual disability, as an IQ of 70 falls above this cutoff.

Attention-deficit/hyperactivity disorder (ADHD), one of the most common neurodevelopmental disorders, is marked by symptoms of inattention, hyperactivity, and impulsivity, and affects $3 \%-8 \%$ of children worldwide. ${ }^{3}$ Both ADHD and ASD are highly heritable childhood-onset disorders, showing significant genetic and behavioral overlaps. ${ }^{4,5}$ The rates of comorbidity between autism and ADHD range from 14\% to $78 \%,{ }^{6}$ in addition, as many as $22 \%-50 \%$ of children with ADHD display elevated clinical symptoms of ASD. ${ }^{7-9}$

According to previous research, both ADHD and ASD are characterized by poor performance on a range of cognitive tasks. ${ }^{10}$ Some studies show similar neurocognitive weaknesses between children with ASD and ADHD, ${ }^{11}$ such as slow processing speed, ${ }^{12}$ dysgraphia, ${ }^{13,14}$ learning disability in written expression, ${ }^{15}$ and deficits in attention, motor control, and perception. ${ }^{16}$

Most previous research highlighting similar defects within specific areas of cognition has utilized IQ tests. Findings indicate that some important features are shared by ASD and ADHD. According to Kaufman, profiles of the Wechsler Scales are characterized by low scores on the freedom from distractibility and processing speed factors, compared with the scores on verbal comprehension and perceptual organization in both ASD and ADHD. It is suggested that children with ASD and ADHD also display poor concentration and poor processing speed. ${ }^{17}$ Nonetheless, these children display an equal ability when compared to typically developing (TD) children in both verbal comprehension and perceptual organization. ${ }^{18}$

While similar IQ profiles have also been found in children with ASD and ADHD, others reported that these children display different profiles. For example, Fried et al found that children with ASD showed significantly more impairment in Working Memory Index (WMI) and PSI (Processing Speed Index) than TD controls, and also showed more impairment in PSI than children with ADHD. ${ }^{19}$ Chiang et al reported that children with ASD showed lower verbal IQ, performance IQ, and full-scale IQ (FSIQ) than children with ADHD. ${ }^{20}$ The inconsistencies within these results may be rooted in the fact that the researchers were including children with ASD in their studies, because it is clear that these two disorders are highly linked. Although the subgroup of ADHD should be taken into account, one study reported no difference on neuropsychological tests including measures of attention, working memory, processing speed, and graphomotor skills among children with autism, ADHD-C (combined type), and ADHD-I (predominantly inattentive) ${ }^{21}$

Based on the literature review, it is clear that inconsistent findings have been reported when it comes to the impairment of working memory and processing speed for children with ADHD and ASD. One possibility for the inconsistency is that the samples included were too heterogeneous. It is possible that gender and different clinical groupings may have also had an effect. Finally, ADHD and ASD are both male-dominated disorders, which means that gender effects could be stronger for these populations. ${ }^{22}$

In the current study, we included boys with ASD and ADHD as the study group, and compared their features with TD boys. We hypothesized that: the boys with high-functioning ASD (HFASD) would mainly be characterized with impairment in Verbal Comprehension Index (VCI), while boys with ADHD would display more impairment in WMI. The present study focused on the similarities and differences in intelligence profiles between children with high-functioning ASD and children with ADHD. The current study aimed 1) to identify the intelligence profiles of Chinese school-aged boys with HFASD and ADHD using the Wechsler Intelligence Scale for Children-IV (WISC-IV) Chinese version; 2) to explore the difference of intelligence between the children with HFASD and ADHD subgroups; and 3) to explore the correlation between the IQ scores and clinical symptoms in the HFASD and ADHD groups separately.

\section{Patients and methods}

\section{Participants}

Thirty-two boys with HFASD, 58 with ADHD, and 39 TD controls took part in this study. All participants were required to have an FSIQ $>70$, and ranged from 6 to 16 years old. The diagnosis was based on DSM- 5 criteria and determined by a medical doctor (MD)-level clinician, under the supervision of an $\mathrm{MD} / \mathrm{PhD}$ Professor (Yasong Du, the corresponding author). Due to the fact that one clinician gave these diagnoses, there were no concerns with inter-rater reliability in diagnoses.

\section{HFASD group}

Boys in this group were aged 6-16 years and diagnosed with HFASD using the DSM-5 criteria at the Department of Child \& Adolescent Psychiatry of Shanghai Mental Health Center, Shanghai Jiaotong University School of Medicine, between 
January, 2014 and December, 2016. Boys in this group all had an FSIQ $\geq 70$ (WISC-IV), and were not taking any antipsychotics at the time of the testing. Children with ADHD were excluded from this group (ADHD was measured by using the result of the Kiddie Schedule for Affective Disorders and Schizophrenia [K-SADS] interview).

\section{ADHD group}

Boys in the ADHD group were 6-16 years old and diagnosed with ADHD by using the DSM-5 at the Department of Child \& Adolescent Psychiatry of Shanghai Mental Health Center, Shanghai Jiaotong University School of Medicine, between January, 2014 and December, 2016. All the boys in this group had FSIQ scores $\geq 70$ (WISC-IV), without taking any central stimulants or psychoactive drugs. Children with ASD were excluded in the ADHD group according to the result of K-SADS (Kiddie-SADS-Present and Lifetime Version [K-SADS-PL]) interview. . $^{23,24}$

The boys in the ADHD group were further divided into three groups based on the classification criteria in DSM-5: predominantly inattentive (ADHD-PI or ADHD-I), predominantly hyperactive-impulsive (ADHD-H), and combined type (ADHD-C).

\section{Typically developing group}

The boys in the TD group were recruited from one primary and one middle school in Xuhui District, Shanghai, China. Children were excluded if they had any type of psychiatric disorder based on the K-SADS-PL. ${ }^{23,24}$

Exclusion criteria included organic diseases and other serious psychiatric disorders, including organic mental disorders, schizophrenia, and other mental disorders; neurodegenerative disorders, traumatic brain injury, or cerebral vascular disease; serious heart, liver, kidney dysfunction, and other major physical illness history or a history of drug dependence.

This study was approved by the Shanghai Mental Health Center Ethics Committee. Parents provided written informed consent for their children. All the children provided written assent by signing their name on the consent form.

\section{Measures}

\section{Wechsler Intelligence scale for children-IV}

The WISC-IV ${ }^{25}$ was introduced in China in 2009 by Zhang. The WISC-IV includes four index scores (Perceptual Reasoning Index, PRI; VCI; WMI; and PSI) and 10 subtests. General Ability Index (GAI) is the comprehensive score of the VCI and PRI. The Cognitive Proficiency Index (CPI) is composed of the WMI and PSI. The VCI includes the vocabulary (VOC), similarities (SIM), and comprehension (COM) subtests; the PRI includes the block design (BD), picture concepts (PCn), and matrix reasoning (MR) subtests; the WMI includes the digit span (DS) and letter-number sequencing (LNS) subtests; and the PSI includes the coding (CD) and symbol search (SS) subtests.

\section{Autism Behavior Checklist}

The Autism Behavior Checklist includes 57 items and five domains, including the sensory, relating, body concept, language, and social self-help domains. ${ }^{26}$ It was introduced in China by Yang et $\mathrm{al}^{27}$ and has been widely used in clinical and scientific research in China. ${ }^{28}$ It can be used with individuals aged from 18 months to 35 years, as a screening tool for ASD.

\section{Conners parent symptom questionnaire}

The Conners Parent Symptom Questionnaire (PSQ) was developed by Conners in 1969 to evaluate the severity of ADHD symptoms in children. Based on the reliability studies in China, the questionnaire demonstrated good reliability (0.932) with urban Chinese children; thus, it demonstrated adequate reliability to use in the current study. ${ }^{29}$ There are 48 items on the PSQ, which yields six factors including conduct problems, learning problems, psychosomatic disorders, impulsivity-hyperactivity, anxiety, and hyperactivity. A higher score indicates more serious behavior problems.

\section{Kiddie-SADS-Present and Lifetime Version}

The K-SADS-PL is a semistructured diagnostic tool, based on the DSM-III-R and DSM-IV, including 1) nonformulary guide checks, 2) screening, 3) a supplementary examination completion list, 4) a diagnostic supplement, 5) a lifetime diagnosis of summary list, and 6) a Global Assessment Scale, which was used to assess the current and previous psychotic episodes of children and adolescents in this study. It has been used extensively in a variety of studies and clinical trials on childhood and adolescent mental disorders, including Chinese children. ${ }^{30}$

\section{Statistical analysis}

Participants' general demographic characteristics were examined and described. All quantitative data were tested for homoscedasticity. Next, to assess the differences among the 
groups, we conducted a one-way analysis of variance, and the Gabriel and Games-Howell test was used for post hoc analysis when the F value was significant. All of the analyses were completed using IBM SPSS version 17.0. ${ }^{31}$

\section{Results}

\section{Descriptive statistics}

The results indicated no statistically significant differences between age among the HFASD, ADHD, and the TD groups $(\mathrm{F}=0.281, P=0.756)$. There was also no difference found between the ADHD-I and ADHD-C subgroups, in both age and PSQ scores (except the impulsivity/hyperactivity score). These results can be found in Table 1 .

\section{Group differences in IQ test}

The FSIQ, GAI, CPI, VCI, and PSI in the HFASD and ADHD groups were all statistically significantly lower than that in the TD group. The VCI in the ASD group was also statistically significantly lower than that in the ADHD group. The WMI was statistically significantly lower in the ADHD group than in the TD group. No significant differences were observed in PRI among the three groups (Table 2).

Looking further, we compared the IQ scores among the HFASD and ADHD subgroups. Due to the small sample in the ADHD-H group ( $n=3)$, we excluded the children with ADHD-H and divided the ADHD group into the remaining ADHD-I and ADHD-C subgroups. Results indicated that the FSIQ, PSI, and CPI scores in the ADHD-I, ADHD-C, and HFASD were all statistically significantly lower than the
TD group. The VCI scores of the ADHD-I, ADHD-C, and HFASD groups were also lower than those in the TD group. Further, the VCI scores among the HFASD group were lower than those in the ADHD-C group. As expected, the WMI scores for both the ADHD-I and ADHD-C subgroups were lower than those in the TD group, while the GAI of the HFASD and ADHD-I was lower than that in the TD group (Table 2; Figure 1).

Finally, we investigated the inner IQ profiles in the HFASD, ADHD, and TD groups, including the difference between the VCI/PRI, WMI/PSI, and GAI/CPI. In the HFASD group, there were no significant differences between the VCI/PRI, WMI/ PSI, or GAI/CPI. In the ADHD group, the VCI score was statistically significantly higher than the PRI score, and the children displayed a lower GAI than CPI. In the ADHD-I and ADHD-C groups, GAI-CPI differences were observed. In the TD group, the VCI was higher than the PRI, WMI was higher than PSI, and GAI was higher than the CPI (Table 3).

\section{Correlation analysis between IQ and clinical manifestation}

We also performed a separate correlation analysis between the IQ scores and clinical symptoms. In the HFASD group, the IQ scores had no correlation with the ASD-related symptoms (Table 4). In the ADHD group, impulsivity/ hyperactivity score was positively correlated with the VCI, WMI, and CPI, the conduct problem score was positively correlated with the CPI, and the learning problem score was negatively correlated with the CPI score (Table 5).

Table I The symptom scores of the ASD and ADHD groups

\begin{tabular}{|c|c|c|c|c|c|}
\hline Groups & Scale/age & Age/subtests & $\mathbf{M} \pm \mathbf{S D}$ & $\mathbf{M} \pm \mathbf{S D}$ & $\mathbf{M} \pm \mathbf{S D}$ \\
\hline ASD group & $A B C$ & Age & $10.31 \pm 3.34$ & & \\
\hline \multirow[t]{6}{*}{$(n=32)$} & & Sensory & $8.96 \pm 5.85$ & - & - \\
\hline & & Social & $12.50 \pm 7.64$ & - & - \\
\hline & & Language & $9.35 \pm 7.34$ & - & - \\
\hline & & Body concept & $9.69 \pm 8.01$ & - & - \\
\hline & & Self-care & $8.42 \pm 5.94$ & - & - \\
\hline & & & ADHD group & ADHD-I group & ADHD-C group \\
\hline ADHD group & $\mathrm{PSQ}$ & Age & $10.38 \pm 2.29$ & $10.55 \pm 2.83$ & $10.28 \pm 2.18$ \\
\hline \multirow[t]{6}{*}{$(n=58)$} & & Conduct problems & $24.84 \pm 6.91$ & $22.69 \pm 5.74$ & $26.17 \pm 6.72$ \\
\hline & & Learning problems & II.59 \pm 2.64 & II.65 \pm 2.74 & $12.03 \pm 2.43$ \\
\hline & & Somatic factor & $6.75 \pm 2.02$ & $7.00 \pm 2.43$ & $6.38 \pm 1.47$ \\
\hline & & Impulsivity/hyperactivity & $|0.2| \pm 2.82$ & $8.80 \pm 2.26$ & $11.45 \pm 2.82 * *$ \\
\hline & & Anxiety & $6.23 \pm 1.88$ & $6.19 \pm 1.81$ & $6.24 \pm 1.88$ \\
\hline & & Hyperactivity index & $24.09 \pm 5.63$ & $22.15 \pm 5.34$ & $25.45 \pm 5.05$ \\
\hline TD group $(n=39)$ & Age & & $|0.72 \pm 2.2|$ & $10.55 \pm 2.39$ & $10.28 \pm 2.19$ \\
\hline
\end{tabular}

Note: $* * p<0.01$.

Abbreviations: ABC, Autism Behavior Checklist; ADHD, attention-deficit/hyperactivity disorder; ADHD-I, ADHD predominantly inattentive; ADHD-C, ADHD combined type; ASD, autism spectrum disorder; M, mean; PSQ, Parent Symptom Questionnaire; TD, typically developing; SD, standard deviation. 
Table 2 The difference of IQ profile among the ASD, ADHD, and TD groups

\begin{tabular}{|c|c|c|c|c|c|c|c|}
\hline $\begin{array}{l}\text { WISC-IV } \\
\text { Chinese version }\end{array}$ & $\begin{array}{l}\text { ASD }(n=32) \\
M \pm \text { SD }\end{array}$ & $\begin{array}{l}\text { ADHD }(n=58) \\
M \pm S D\end{array}$ & $\begin{array}{l}\text { ADHD-I }(\mathrm{N}=26) \\
\mathrm{M} \pm \mathrm{SD}\end{array}$ & $\begin{array}{l}\text { ADHD-C }(\mathrm{N}=29) \\
\mathrm{M} \pm \mathrm{SD}\end{array}$ & $\begin{array}{l}\text { TD }(n=39) \\
M \pm S D\end{array}$ & $\begin{array}{l}\text { Post hoc } \\
\text { comparisons }\end{array}$ & $\begin{array}{l}\text { Post hoc } \\
\text { comparisons }\end{array}$ \\
\hline Similarity & $10.72 \pm 4.105$ & $12.09 \pm 2.69$ & $11.46 \pm 1.94$ & $|2.38 \pm 3.0|$ & $13.95 \pm 2.03$ & $\begin{array}{l}\mathrm{ADHD}<\mathrm{TD} * * \\
\mathrm{ASD}<\mathrm{TD} * *\end{array}$ & $\begin{array}{l}\text { ADHD-I }<\text { TD**; } \\
\text { ASD }<\text { TD** }\end{array}$ \\
\hline Vocabulary & $8.13 \pm 4.046$ & $11.17 \pm 3.13$ & $10.81 \pm 3.14$ & $11.17 \pm 3.14$ & $12.74 \pm 2.49$ & ASD $<\mathrm{TD}^{* *}$ & $\begin{array}{l}\text { ASD }<\text { ADHD-I*; } \\
\text { ASD }<\text { ADHD-C**; } \\
\text { ASD }<\text { TD** }\end{array}$ \\
\hline Comprehension & $8.03 \pm 4.246$ & $10.36 \pm 2.82$ & $9.69 \pm 2.83$ & $10.62 \pm 2.67$ & $12.05 \pm 3.15$ & $\begin{array}{l}\mathrm{ADHD}<\mathrm{TD}^{*} ; \\
\mathrm{ASD}<\mathrm{TD}^{* *}\end{array}$ & $\begin{array}{l}\text { ASD }<\text { ADHD-C*; } \\
\text { ASD }<\text { TD**; } \\
\text { ADHD-I }<\text { TD* }\end{array}$ \\
\hline Block design & $|2.3| \pm 4.02$ & $11.17 \pm 2.82$ & $10.46 \pm 2.44$ & $11.79 \pm 3.09$ & $11.77 \pm 2.66$ & ns & ns \\
\hline Picture concept & $8.88 \pm 3.26$ & $9.31 \pm 2.43$ & $9.00 \pm 2.64$ & $9.62 \pm 2.35$ & $10.15 \pm 2.31$ & ns & ns \\
\hline Matrix & $9.56 \pm 3.66$ & $10.4 \mid \pm 2.52$ & $10.38 \pm 2.12$ & $10.34 \pm 2.94$ & $11.05 \pm 2.55$ & ns & ns \\
\hline Digit span & $8.91 \pm 3.75$ & $8.07 \pm 2.707$ & $7.77 \pm 2.60$ & $8.14 \pm 2.79$ & $9.46 \pm 2.45$ & ns & ns \\
\hline Letter-number & $8.5 \pm 4.19$ & $8.81 \pm 2.47$ & $8.35 \pm 2.62$ & $9.07 \pm 2.19$ & $10.87 \pm 2.79$ & $\begin{array}{l}\mathrm{ADHD}<\mathrm{TD} * * \\
\mathrm{ASD}<\mathrm{TD} * *\end{array}$ & $\begin{array}{l}\text { ADHD-I }<\text { TD*; } \\
\text { ASD }<\text { TD* }\end{array}$ \\
\hline Coding & $7.66 \pm 3.17$ & $9.09 \pm 3.40$ & $8.38 \pm 2.83$ & $9.83 \pm 3.78$ & $11.46 \pm 3.19$ & $\begin{array}{l}\mathrm{ADHD}<\mathrm{TD} * * \\
\mathrm{ASD}<\mathrm{TD} * *\end{array}$ & $\begin{array}{l}\text { ADHD-I }<\text { TD**; } \\
\text { ASD }<\text { TD** }\end{array}$ \\
\hline Symbol search & $8.4 I \pm 3.17$ & $8.88 \pm 2.64$ & $8.81 \pm 2.73$ & $9.03 \pm 2.72$ & $11.59 \pm 3.12$ & $\begin{array}{l}\mathrm{ADHD}<\mathrm{TD}^{* *} \\
\mathrm{ASD}<\mathrm{TD}^{* *}\end{array}$ & $\begin{array}{l}\text { ADHD-I }<\text { TD**; } \\
\text { ADHD-C }<\text { TD**; } \\
\text { ASD }<\text { TD** }\end{array}$ \\
\hline $\mathrm{VCl}$ & $94.34 \pm 22.67$ & $107.12 \pm 14.21$ & $103.73 \pm|2.0|$ & $108.24 \pm 15.23$ & $117.36 \pm 13.32$ & $\begin{array}{l}\text { ADHD }<\text { TD**; } \\
\text { ASD }<\mathrm{TD}^{* *} ; \\
\text { ASD }<\text { ADHD** }\end{array}$ & $\begin{array}{l}\text { ADHD-I }<\text { TD*; } \\
\text { ASD }<\text { TD**; } \\
\text { ASD }<\text { ADHD-C* }\end{array}$ \\
\hline PRI & $100.34 \pm 19.49$ & $101.55 \pm 11.02$ & $99.42 \pm 9.37$ & $103.28 \pm 12.60$ & $105.97 \pm 10.62$ & ns & ns \\
\hline WMI & $93.28 \pm 19.67$ & $90.5 \pm 13.32$ & $88.62 \pm 13.60$ & $91.55 \pm 12.48$ & $100.69 \pm 12.94$ & $\mathrm{ADHD}<\mathrm{TD}^{* *}$ & $\begin{array}{l}\text { ADHD-I }<\text { TD }^{* *} ; \\
\text { ADHD-C }<\text { TD* }^{*}\end{array}$ \\
\hline PSI & $88.8 I \pm 17.01$ & $94.14 \pm 13.59$ & $91.73 \pm 13.42$ & $96.59 \pm 14.03$ & $108.28 \pm 15.22$ & $\begin{array}{l}\mathrm{ADHD}<\mathrm{TD} * * \\
\mathrm{ASD}<\mathrm{TD}^{* *}\end{array}$ & $\begin{array}{l}\text { ADHD-I }<\text { TD**; } \\
\text { ADHD-C }<\text { TD*; } \\
\text { ASD }<\text { TD** }\end{array}$ \\
\hline GAI & $97.75 \pm 22.44$ & $105.17 \pm 11.85$ & $103.00 \pm 10.97$ & $106.34 \pm 12.82$ & $113.82 \pm 11.26$ & $\begin{array}{l}\text { ADHD }<\text { TD*; } \\
\text { ASD }<\text { TD** }\end{array}$ & $\begin{array}{l}\text { ADHD-I }<\text { TD*; } \\
\text { ASD }<\text { TD** }\end{array}$ \\
\hline FSIQ & $94.44 \pm 20.53$ & $99.26 \pm 11.79$ & $96.08 \pm 10.53$ & $101.28 \pm 12.65$ & $111.38 \pm 11.29$ & $\begin{array}{l}\mathrm{ADHD}<\mathrm{TD} * * \\
\mathrm{ASD}<\mathrm{TD}^{* *}\end{array}$ & $\begin{array}{l}\text { ADHD-I }<\text { TD**; } \\
\text { ADHD-C }<\text { TD*; } \\
\text { ASD }<\text { TD** }\end{array}$ \\
\hline
\end{tabular}

Notes: $* * P<0.01, * P<0.05$

Abbreviations: ADHD, attention-deficit/hyperactivity disorder; ADHD-I, ADHD predominantly inattentive; ADHD-C, ADHD combined type; ASD, autism spectrum disorder; CPI, Cognitive Proficiency Index; FSIQ, Full-Scale Intelligence Quotient; GAI, General Ability Index; ns, no significant difference; PRI, Perceptual Reasoning Index; PSI, Processing Speed Index; SD, standard deviation; TD, typically developing; VCI, Verbal Comprehension Index; WISC-IV, Wechsler Intelligence Scale for Children-IV; WMI, Working Memory Index.

\section{Discussion}

Though HFASD is classified as a pervasive developmental disorder and ADHD is a neurodevelopmental disorder, both are common in children and adolescents. Researchers have suggested that children with HFASD and ADHD suffer from cognitive function impairments. ${ }^{5}$ Intelligence is a comprehensive presentation of cognitive function. Previous studies have investigated the intelligence profiles of children with HFASD and ADHD, but few studies compared the difference of intelligence between children with HFASD and ADHD. The results of these studies have been inconsistent. In this study, we examined and compared the intelligence profiles of Chinese school-aged boys with HFASD, ADHD, and TD children.

\section{Intellectual functioning within boys with HFASD}

Children with HFASD scored lower on the VCI, PSI, GAI, CPI, and FSIQ, indicating that children with HFASD may be impaired in verbal communication and processing speed as compared to their TD peers. This is a logical finding because language difficulty and sensory processing deficits are both typical features in children with ASD ${ }^{31}$ In our study, children with ASD showed impairments in all the subtests within the VCI. Children typically performed the best on the SIM subtest, followed by VOC, and then the COM subtest. This is consistent with the findings from Zayat et al, who also demonstrated that children with ASD showed the 


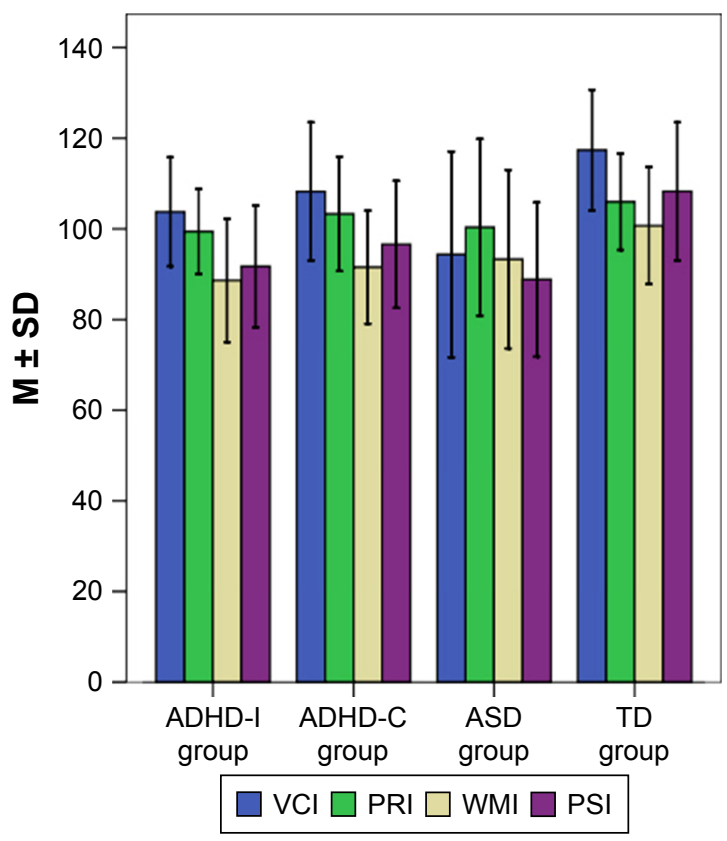

Figure I IQ profile in the ADHD, ASD and TD groups.

Abbreviations: ADHD, attention-deficit/hyperactivity disorder; ADHD-I, ADHD predominantly inattentive; ADHD-C, ADHD combined type; ASD, autism spectrum disorder; TD, typically developing; IQ, intelligence quotient; $\mathrm{VCl}$, Verbal Comprehension Index; PRI, Perceptual Reasoning Index; WMI, Working Memory Index; PSI, Processing Speed Index; M, mean; SD, standard deviation.

same pattern on the VCI subtests of the WISC-IV. ${ }^{32}$ It is likely that the COM subtest would be the most difficult, as it requires a higher capability in language as well as better understanding of social rules.

Our finding of children with HFASD displaying deficits in PSI is in accordance with the previous findings. ${ }^{12,33}$ PSI assesses visual information processing and visual memory. As we know, children with ASD suffer from sensory processing deficits, and sensory processing impairments are highly prevalent in children with HFASD according to previous researchers. ${ }^{31}$ Oliveras-Rentas et al, proposed that due to the task impurity of PSI, it is unclear whether this relative weakness reflects actual "cognitive" processing speed, motor speed difficulties, or the two combined, ${ }^{34}$ as some studies using tasks free from motor demands found that PSI was not impaired. ${ }^{35}$

\section{Intelligence profiles of boys with ADHD}

Due to the fact that the CPI is composed of the PSI and WMI index scores and children with ADHD typically display weaknesses in these areas on the WISC-IV, it was expected that children with ADHD would display a lower CPI. Working memory is one of the core deficits in ADHD, which has been frequently reported since the first report of Barkley, ${ }^{36}$ which is regarded as a part of executive functioning. ${ }^{37}$ Many studies since have confirmed a working memory defect in children with ADHD. ${ }^{38}$

In comparing the ADHD-I and ADHD-C subgroups of ADHD, both groups shared similar features on the WISC-IV. Our findings indicated that the "cognitive efficiency" index is lower in children with ADHD, which is consistent with the previous findings. ${ }^{39}$ Thaler's study suggested that children with ADHD showed different profiles on the WISC-IV, including a lower PSI score (more common in ADHD-I children) and lower WMI scores, which are both associated with impaired behavioral functioning. ${ }^{40}$

\section{The similarities and differences between children with HFASD and ADHD}

Based on our results, children with HFASD and ADHD both displayed impairments in PSI. This is similar to the findings by Oliveras-Rentas et al, ${ }^{34}$ who found a weakness in PSI within high-functioning autism as well as Yang et al, who found weaknesses in PSI in children with ADHD. ${ }^{41}$ Deficits in PSI have been reported in neurodevelopmental disorders, such as ASD, ADHD, and children with learning disabilities. ${ }^{12}$ As we know, PSI has the lowest factor loading on intelligence tests, which may indicate that PSI is the most independent factor in IQ. Additionally, CD and SS require various abilities such as visual seeking, eye-hand coordination, and fine-motor skills. Kaufman has pointed out that PSI can be negatively impacted by these neurological variables, which are common in both children with ASD and ADHD. Furthermore, in Thaler's

Table 3 The inner differences in the ASD, ADHD, and TD groups

\begin{tabular}{llll}
\hline Groups & $\begin{array}{l}\text { VCI-PRI comparison, } \\
\text { paired } t \text {-test/P-value }\end{array}$ & WMI-PSI comparison, t/p & GAI-CPI comparison, t/p \\
\hline ASD group $(n=32)$ & $11.135 / 0.261$ & $0.972 / 0.335$ & $1.545 / 0.127$ \\
ADHD group $(n=58)$ & $2.358 / 0.020$ & $-1.456 / 0.148$ & $6.036 /<0.001$ \\
ADHD-I group $(n=26)$ & $1.442 / 0.156$ & $-0.832 / 0.410$ & $4.892 /<0.001$ \\
ADHD-C group $(n=29)$ & $1.353 / 0.182$ & $-1.444 / 0.154$ & $3.622 / 0.001$ \\
TD group $(n=39)$ & $4.173 /<0.001$ & $-2.373 / 0.020$ & $2.987 / 0.004$ \\
\hline
\end{tabular}

Abbreviations: ADHD, attention-deficit/hyperactivity disorder; ADHD-I, ADHD predominantly inattentive; ADHD-C, ADHD combined type; ASD, autism spectrum disorder; CPI, Cognitive Proficiency Index; GAI, General Ability Index; PRI, Perceptual Reasoning Index; PSI, Processing Speed Index; TD, typically developing; VCI, Verbal Comprehension Index; WMI, Working Memory Index. 
Table 4 The correlation analysis among the IQ scores and clinical symptoms in the ASD group

\begin{tabular}{lllllllll}
\hline ABC & & FSIQ & VCI & PRI & WMI & PSI & GAI & CPI \\
\hline Sensory & $r$ & -0.12 & -0.165 & $-0.00 I$ & -0.229 & 0.059 & -0.308 & 0.048 \\
& $P$ & 0.558 & 0.421 & 0.995 & 0.26 & 0.776 & 0.126 & 0.818 \\
Social & $r$ & -0.047 & -0.198 & 0.113 & -0.103 & 0.186 & -0.076 & 0.074 \\
& $P$ & 0.818 & 0.333 & 0.583 & 0.616 & 0.363 & 0.714 & 0.719 \\
Language & $r$ & $-0.05 I$ & -0.124 & 0.012 & -0.062 & 0.242 & -0.076 & 0.076 \\
& $P$ & 0.804 & 0.547 & 0.954 & 0.763 & 0.233 & 0.713 & 0.712 \\
Body & $r$ & 0.145 & 0.085 & 0.130 & 0.037 & $0.28 I$ & -0.055 & 0.322 \\
& $P$ & 0.479 & 0.678 & 0.526 & 0.859 & 0.165 & 0.788 & 0.109 \\
Selfcare & $r$ & 0.021 & 0.046 & 0.005 & -0.161 & 0.176 & -0.057 & 0.084 \\
& $P$ & 0.92 & 0.825 & 0.982 & 0.432 & 0.39 & 0.782 & 0.683 \\
\hline
\end{tabular}

Abbreviations: ABC, Autism Behavior Checklist; ASD, autism spectrum disorder; CPI, Cognitive Proficiency Index; FSIQ, Full-Scale IQ; GAI, General Ability Index; IQ, intelligence quotient; PRI, Perceptual Reasoning Index; PSI, Processing Speed Index; VCl, Verbal Comprehension Index; WMI, Working Memory Index.

finding, processing speed is associated with inattention, which is one of the featured ADHD profiles that had lower PSI scores. ${ }^{40}$

Further, as we know, verbal difficulty is often a core deficit in children with HFASD. In the DSM-IV, having a language defect is one of the core diagnostic criteria. Though the DSM-5 does not acknowledge this within the diagnostic criteria, it is still clinically relevant and evident within many children with ASD. Our results indicated that verbal COM was impaired in both the HFASD and ADHD-I groups. Koyama et al reported that children with HFPDD-NOS (highfunctioning pervasive developmental disorder not otherwise specified) scored significantly lower on VOC and COM but significantly higher on $\mathrm{BD}$ than the ADHD children using WISC-III; our findings are similar to Koyama's report when

Table 5 The correlation analysis among the IQ scores and clinical symptoms in the ADHD group

\begin{tabular}{|c|c|c|c|c|c|c|c|}
\hline PSQ & FSIQ & VCl & PRI & WMI & PSI & GAI & CPI \\
\hline & $r 0$ & & & & & & \\
\hline & & & & & & 58 & .041 \\
\hline & $r-0.225$ & -0.162 & -0.108 & -0.138 & -0.202 & -0.126 & -0.269 \\
\hline & & & & & & & \\
\hline & $r-0.103$ & -0.066 & -0.024 & 0.017 & -0.2 & -0.131 & -0.028 \\
\hline & $P 0.44 I$ & 0.625 & 0.856 & & & 329 & 0.833 \\
\hline & & & & & & & \\
\hline & $P 0.074$ & 0.043 & 0.693 & 0.045 & 0.376 & .195 & 0.027 \\
\hline \multirow[t]{2}{*}{ Anxiety } & 208 & -0.113 & -0.2 & -0 . & -0.085 & -0.228 & -0.106 \\
\hline & $P 0.118$ & 0.400 & 0.057 & 0.29 & 0.525 & 0.085 & 0.428 \\
\hline & r 0.116 & 0.059 & 0.113 & 0.172 & 0.051 & 0.037 & 0.228 \\
\hline & & 0.661 & 0.397 & 0.196 & 0.704 & 0.785 & 0.086 \\
\hline
\end{tabular}

Abbreviations: ADHD, attention-deficit/hyperactivity disorder; CPI, Cognitive Proficiency Index; FSIQ, Full-Scale IQ; GAI, General Ability Index; IQ, intelligence quotient; PRI, Perceptual Reasoning Index; PSI, Processing Speed Index; PSQ, Conners Parent Symptom Questionnaire; $\mathrm{VCl}$, Verbal Comprehension Index; WMI, Working Memory Index. it comes to VOC and COM, though Koyama did not make distinctions between the ADHD groups. ${ }^{42}$

As has been discussed before, working memory is one of the main deficits in ADHD. We did not find evidence of working memory weaknesses in children with ASD in this study, though lower working memory scores have been reported in other studies..$^{43}$ In our study, we used digital and letter number tests as the indicators of working memory, which may not be sensitive enough to detect working memory defects in children with ASD. ${ }^{44}$ Children with HFASD and ADHD showed similar abilities when it comes to perceptual reasoning to TD children in the current study, which is also consistent with the previous findings. ${ }^{18}$

\section{The correlation between IQ scores and clinical symptoms}

Through this study we did not find any relationship between IQ performance and clinical manifestation in children with HFASD. We did, however, find that VCI, WMI, and CPI were positively related to children's behavior level of hyperactivity in the ADHD group. Additionally, conduct problems were positively correlated with the CPI score, and, learning problems were negatively correlated with the CPI, which needs further investigation. This also indicates the importance of investigating the subgroup of hyperactivity/ impulsivity subgroup within children with ADHD.

\section{Conclusion}

We found that both children with ADHD and HFASD have difficulties in processing speed, which supports the idea that a PSI defect is more related to neurodevelopmental disorders. Based on diagnostic criteria, children with HFASD may have language deficits, which is consistent with its core defect, while children with ADHD typically display working memory difficulties, which is more consistent with an attention defect. All of these findings will add to the research in assessing children with ASD and ADHD in clinical setting. In addition, it is possible that intellectual impairments may be addressed in clinical practice, for instance utilizing PSI training to assist in enhanced cognitive functioning.

\section{Limitations}

First, in this study, we did not compare behavioral differences between children with ASD and ADHD. As HFASD and ADHD are highly comorbid with each other, it may be important to further evaluate behavioral differences, which may impact symptoms within ASD and ADHD. Though the authors made efforts to ensure that there were no overlapping 
diagnoses (eg, children with ASD in the ADHD group or children with ADHD in the ASD group), these two disorders are highly comorbid and there is still a possibility that overlap existed. Second, we divided the children with ADHD into subgroups, but there were only three children in the ADHD-H group, so this group was too small to analyze and had to be excluded. Future studies should include a larger sample size so that subgroups do not become too small when the sample is divided. Further, the age range is from 6 to 16 in the current study, which includes a broad range of development. Although the IQ test utilized was standardized for this age range, a longitudinal analysis is still needed to clarify the variance from the different developmental stages. More age-homogeneous samples are needed in further studies in order to ensure that development does not greatly impact the maturation of the processes examined in this study. Finally, we did not include girls in this study. These are all future directions.

\section{Acknowledgments}

We would like to thank all of the children and their families who participated in the current study. This study was supported by the SHSMU-ION; Research Center for Brain Disorders, Grant number: 2015NKX005.

\section{Authors' contributions}

Dr GL and Dr WJ contributed equally to this manuscript including data collection, analysis, and writing the manuscript. Dr YD worked on the design part of this work and is the corresponding author of this manuscript. Dr KR revised this manuscript several times, addressing the language and format, and also rewrote some paragraphs in the introduction and discussion sections. All authors contributed toward data analysis, drafting and revising the paper and agree to be accountable for all aspects of the work.

\section{Disclosure}

The authors report no conflicts of interest in this work.

\section{References}

1. Christensen DL, Baio J, Braun KVN, et al. Prevalence and characteristics of autism spectrum disorder among children aged 8 years-autism and developmental disabilities monitoring network, 11 sites, United States, 2012. MMWR Surveill Summ. 2016;65(3):1-23.

2. Lincoln AJ, Allen MH, Kilman A. The assessment and interpretation of intellectual abilities in people with autism. In: Learning and Cognition in Autism. US: Springer; 1995:89-117.

3. Steinhausen HC. The heterogeneity of causes and courses of attentiondeficit/hyperactivity disorder. Acta Psychiatr Scand. 2009;120(5): 392-399.

4. Ronald A, Happe F, Plomin R. A twin study investigating the genetic and environmental aetiologies of parent, teacher and child ratings of autisticlike traits and their overlap. Eur Child Adolesc Psychiatry. 2008;17(8): 473-483.
5. Rommelse NN, Geurts HM, Franke B, Buitelaar JK, Hartman CA. A review on cognitive and brain endophenotypes that may be common in autism spectrum disorder and attention-deficit/hyperactivity disorder and facilitate the search for pleiotropic genes. Neurosci Biobehav Rev. 2011;35(6):1363-1396.

6. Gargaroa BA, Rinehartb NJ, Bradshawa JL, Tongeb BJ, Sheppard DM. Autism and ADHD: how far have we come in the comorbidity debate? Neurosci Biobehav Rev. 2011;35(5):1081-1088.

7. Reiersen AM, Constantino JN, Volk HE, Todd RD. Autistic traits in a population-based ADHD twin sample. J Child Psychol Psychiatry. 2007;48(5):464-472.

8. Kochhar P, Batty MJ, Liddle EB, et al. Autistic spectrum disorder traits in children with attention deficit hyperactivity disorder. Child Care Health Dev. 2011;37(1):103-110.

9. van der Meer JMJ, Oerlemans AM, van Steijn DJ, et al. Are autism spectrum disorder and attention-deficit/hyperactivity disorder different manifestations of one overarching disorder? Cognitive and symptom evidence from a clinical and population-based sample. J Am Acad Child Adolesc Psychiatry. 2012;51(11):1160-1172.e3.

10. Corbett BA, Constantine LJ, Hendren R, Rocke D, Ozonoff S. Examining executive functioning in children with autism spectrum disorder, attention deficit hyperactivity disorder and typical development. Psychiatry Res. 2009;166(2-3):210-222.

11. Happe F, Booth R, Charlton R, Hughes C. Executive function deficits in autism spectrum disorders and attention-deficit/hyperactivity disorder: examining profiles across domains and ages. Brain Cogn. 2006; 61(1):25-39.

12. Calhoun SL, Mayes SD. Processing speed in children with clinical disorders. Psychol Schs. 2005;42(4):333-343.

13. Mayes SD, Calhoun SL. Similarities and differences in WISC-III profiles: support for subtest analysis in clinical referrals. Clin Neuropsychol. 2004;18(4):559-572.

14. Mayes SD, Calhoun SL. Learning, attention, writing, and processing speed in typical children and children with ADHD, autism, anxiety, depression, and oppositional-defiant disorder. Child Neuropsychol. 2007;13(6):469-493.

15. Mayes SD, Calhoun SL. Frequency of reading, math, and writing disabilities in children with clinical disorders. Learn Individ Differ. 2006; 16:145-147.

16. Sturm H, Fernell E, Gillberg C. Autism spectrum disorders in children with normal intellectual levels: associated impairments and subgroups. Dev Med Child Neurol. 2004;46(7):444-447.

17. Kaufman AS. Assessment: The Wechsler Scales and Learning Disabilities. J Learn Disabil. 1981;14(7):397-398.

18. Ashwood KL, Tye C, Azadi B, Cartwright S, Asherson P, Bolton P. Brief report: adaptive functioning in children with ASD, ADHD and ASD + ADHD. J Autism Dev Disord. 2015;45(7):2235-2242.

19. Fried R, Joshi G, Bhide P, et al. A study of the neuropsychological correlates in adults with high functioning autism spectrum disorders. Acta Neuropsychiatrica. 2016;28(5):286-295.

20. Chiang HL, Chen YJ, Lin HY, Tseng WYI, Gau SS. Disorder-specific alteration in white matter structural property in adults with autism spectrum disorder relative to adults with ADHD and adult controls. Hum Brain Mapp. 2017;38(1):384-395.

21. Mayes SD, Calhoun SL, Mayes RD, Molitoris S. Autism and ADHD: overlapping and discriminating symptoms. Res Autism Spectr Disord. 2012;6(1):277-285.

22. Zahnwaxler C, Shirtcliff EA, Marceau K. Disorders of childhood and adolescence: gender and psychopathology. Ann Rev Clin Psychol. 2008;4(4):275-303.

23. Birmaher B, Ehmann M, Axelson DA, et al. Schedule for affective disorders and schizophrenia for school-age children (K-SADS-PL) for the assessment of preschool children-a preliminary psychometric study. J Psychiatr Res. 2009;43(7):680-686.

24. Kaufman J, Birmaher B, Brent D, et al. Schedule for affective disorders and schizophrenia for school-age children-present and lifetime version (K-SADS-PL): initial reliability and validity data. J Am Acad Child Adolesc Psychiatry. 1997;36(7):980-988. 
25. Zhang HC. The revision of WISC-IV Chinese version. Psychol Sci. 2009; 32:1177-1179.

26. Krug DA, Arick J, Almond P. Behavior checklist for identifying severely handicapped individuals with high levels of autistic behavior. J Child Psychol Psychiatry. 1980;21(3):221-229.

27. Yang XL, Huang YQ, Jia MX, Chen SK. Test report of Autism Behavior Checklist. Chin Mental Health J. 1993;7(6):279-280.

28. Jiang LX, Li GZ, Du YS. Comparison of screening tools of Autism Spectrum Disorder. Chin J Nerv Ment Dis. 2015;41(3):189-192.

29. Fan J, Du YS, Wang LW. The norm and reliability of the Conners Parent Symptom Questionnaire in Chinese urban children. Shanghai Arch Psychiatr. 2005;17(6):321-323. Chinese.

30. Gau SS, Chong MY, Chen TH, Cheng AT. A 3-year panel study of mental disorders among adolescents in Taiwan. Am J Psychiatry. 2005;162(7):1344-1350.

31. Costa RC, Lampreia C. Findings on sensory deficits in autism: Implications for understanding the disorder. Psychol Neurosci. 2012;5: 231-237.

32. Zayat M, Kalb L, Wodka EL. Brief report: performance pattern differences between children with autism spectrum disorders and attention deficit-hyper-activity disorder on measures of verbal intelligence. J Autism Dev Disord. 2011;41:1743-1747.

33. Mouga S, Café C, Almeida J, Marques C, Duque F, Oliveira G. Intellectual profiles in the autism spectrum and other neurodevelopmental disorders. J Autism Dev Disord. 2016;46(9):2940-2955.

34. Oliveras-Rentas RE, Kenworthy L, Roberson III RB, Martin A, Wallace GL. WISC-IV profile in high-functioning autism spectrum disorders: impaired processing speed is associated with increased autism communication symptoms and decreased adaptive communication abilities. J Autism Dev Disord. 2012;42(5):655-664.

35. Scheuffgen K, Happe' F, Anderson M, Frith U. High “intelligence”, low "IQ"? Speed of processing and measured IQ in children with autism. Dev Psychopathol. 2000;12(1):83-90.

36. Barkley RA. Behavioral inhibition, sustained attention, and executive functions: constructing a unifying theory of ADHD. Psychol Bull. 1997; 121(1):65-94.
37. Biederman J, Monuteaux MC, Doyle AE, et al. Impact of executive function deficits and attention-deficit/hyperactivity disorder (ADHD) on academic outcomes in children. J Consult Clin Psychol. 2004; 72(5):757-766.

38. Shimoni M, Engel-Yeger B, Tirosh E. Executive dysfunctions among boys with Attention Deficit Hyperactivity Disorder (ADHD): performance-based test and parents report. Res Dev Disabil. 2012;33(3): $858-865$.

39. Jiang WQ, Li Y, Du YS, Fan J. Cognitive Deficits feature of male with attention deficit hyperactivity disorder - based on the Study of WISC-IV. Afr J Psychiatry. 2013;18(2).

40. Thaler NS, Bello DT, Etcoff LM. WISC-IV profiles are associated with differences in symptomatology and outcome in children with ADHD. J Atten Disord. 2013;17(4):291-301.

41. Yang P, Cheng CP, Chang CL, Liu TL, Hsu HY, Yen CF. Wechsler Intelligence Scale for Children 4th edition-Chinese version index scores in Taiwanese children with attention-deficit/hyperactivity disorder. Psychiatry Clin Neurosci. 2013;67(2):83-91.

42. Koyama T, Tachimori H, Osada H, Kurita H. Cognitive and symptom profiles in high-functioning pervasive developmental disorder not otherwise specified and attention-deficit/hyperactivity disorder. J Autism Dev Disord. 2006;36(3):373-380.

43. Barendse EM, Hendriks MPH, Jansen JFA, et al. Working memory deficits in high-functioning adolescents with autism spectrum disorders: neuropsychological and neuroimaging correlates. J Neurodev Disord. 2013;5(1):14.

44. Nakahachi T, Iwasw M, Takahashi H, et al. Discrepancy of performance among working memory-related tasks in autism spectrum disorders are caused by task characteristics, apart from working memory, which could interfere with task execution. Psychiatry Clin Neurosci. 2006;60(3):312-318.
Neuropsychiatric Disease and Treatment

\section{Publish your work in this journal}

Neuropsychiatric Disease and Treatment is an international, peerreviewed journal of clinical therapeutics and pharmacology focusing on concise rapid reporting of clinical or pre-clinical studies on a range of neuropsychiatric and neurological disorders. This journal is indexed on PubMed Central, the 'PsycINFO' database and CAS,

\section{Dovepress}

and is the official journal of The International Neuropsychiatric Association (INA). The manuscript management system is completely online and includes a very quick and fair peer-review system, which is all easy to use. Visit http://www.dovepress.com/testimonials.php to read real quotes from published authors. 